Louisiana State University

LSU Digital Commons

$5-1-2016$

\title{
Long-term shifts in the patterns and underlying processes of plant associations in Wisconsin forests
}

\author{
Daijiang Li \\ University of Wisconsin-Madison \\ Donald Waller \\ University of Wisconsin-Madison
}

Follow this and additional works at: https://digitalcommons.Isu.edu/biosci_pubs

\section{Recommended Citation}

Li, D., \& Waller, D. (2016). Long-term shifts in the patterns and underlying processes of plant associations in Wisconsin forests. Global Ecology and Biogeography, 25 (5), 516-526. https://doi.org/10.1111/ geb.12432

This Article is brought to you for free and open access by the Department of Biological Sciences at LSU Digital Commons. It has been accepted for inclusion in Faculty Publications by an authorized administrator of LSU Digital Commons. For more information, please contact ir@lsu.edu. 


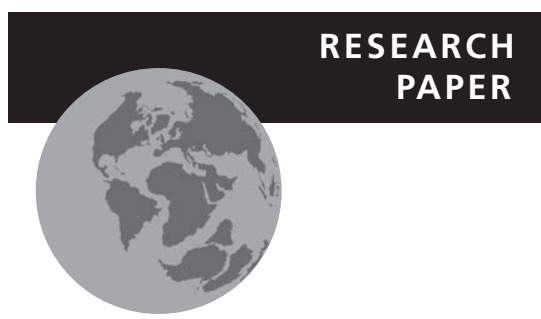

\section{Long-term shifts in the patterns and underlying processes of plant associations in Wisconsin forests}

Daijiang Li* and Donald Waller

Department of Botany, University of Wisconsin-Madison, 430 Lincoln Drive,

Madison, WI 53706, USA
${ }^{*}$ Correspondence: Daijiang Li, Department of Botany, University of Wisconsin-Madison, 430 Lincoln Drive, Madison, WI 53706, USA. E-mail: daijianglee@gmail.com

\begin{abstract}
Aim Plant species co-occur within communities in response to variation in environmental conditions, limited species dispersal and biotic interactions. We used surveys and resurveys of the same sites of three temperate forest plant communities to study patterns of association between co-occurring species pairs and to infer how these mechanisms contribute to community assembly. Our goal was to compare these forces among communities occupying more and less disturbed landscapes and examine how these have changed over the last 50 years.
\end{abstract}

Location Wisconsin, USA.

Methods We resurveyed 266 sites first surveyed in the 1950 s to assess the patterns and dynamics of co-occurrence among understorey plant species in three community types. We then used checkerboard scores, null models and a newly developed framework to infer the mechanisms likely to have driven community assembly. Finally, we compared these across the three communities and two time periods.

Results Species co-occur less often than expected in all three vegetation types and both periods. We detected high fractions of aggregated and segregated species pairs (up to $14 \%$ and $17 \%$, respectively). In the fragmented southern upland forests and central sand plains, both biotic interactions and dispersal limitation may play important roles in community assembly with inferred dispersal limitation becoming more important since the 1950s. In the more continuous and intact northern upland forests, environmental filtering and biotic interactions appear to dominate community dynamics with little change over time. Aggregated and segregated species pairs made a similar contribution to our ability to infer these mechanisms.

Main conclusions Environmental filtering, biotic interactions and dispersal limitation all appear to affect plant community structure in both time periods. However, the influence of dispersal limitation seems to be increasing in more fragmented forest landscapes, portending shifts in community composition and dynamics. Because aggregated and segregated species pairs may be shaped by similar processes both can be used to infer processes of community assembly.

\section{Keywords}

Biotic interactions, community assembly rules, dispersal limitation, environmental filtering, null models, species co-occurrence.

\section{INTRODUCTION}

What factors drive the structure, composition and dynamics of natural communities? There is an emerging consensus that both deterministic and stochastic factors affect community species composition (Gravel et al., 2006). Deterministic factors include habitat filtering and species interactions (e.g. competition; Chase \& Leibold, 2003). Stochastic factors include dispersal, ecological drift and random extinction and speciation (Hubbell, 2001). Among these factors, environmental filtering, species interactions and dispersal limitation are considered to be the most important (Götzenberger et al., 2012). The fundamental 
niche of a species determines the environmental conditions within which it can survive and maintain itself. Dispersal ability, in turn, delimits the habitats that a species can reach within its fundamental niche. Finally, biotic interactions with other species within those habitats shape the realized niche of a species. Because these three mechanisms operate simultaneously, they are hard to distinguish. Consequently, their relative importance in shaping plant community assembly remains controversial.

Analyses of species co-occurrence patterns have played an important role in inferring the drivers of community assembly (Diamond, 1975; Gotelli, 2000). Early studies focused on distinguishing deterministic forces such as competition from random forces using null models that analysed patterns of species co-occurrence among sites (Diamond, 1975; Gotelli \& McCabe, 2002). These efforts, however, led to controversy over which null models were most appropriate and what could be inferred from overall departures from randomness (Gotelli, 2000). From this controversy emerged a better understanding of the limitations of simple approaches that do not distinguish competition from other forces driving species associations including dispersal limitation and environmental filtering. This, in turn, has led to new efforts to detect and untangle the forces that drive community assembly.

Most commonly, ecologists characterize overall patterns of co-occurrence among species by tallying community-wide indices like the checkerboard score (C-score), using the average pair-wise occurrence of checkerboard units (Stone \& Roberts, 1990). A checkerboard unit is a $2 \times 2$ site or quadrat by species matrix wherein both species occur once but in different locations, for example

$\left[\begin{array}{ll}1 & 0 \\ 0 & 1\end{array}\right]$ or $\left[\begin{array}{ll}0 & 1 \\ 1 & 0\end{array}\right]$.

The significance of C-scores is usually evaluated by comparing the observed value with C-scores generated by randomized null models in which row and column sums are fixed (fixed-fixed null models; SIM9 in Gotelli, 2000). Strong environmental filtering and competition between species will often result in communities where species co-occur more or less than expected by chance (reflecting aggregation and segregation, respectively). Alternatively, one expects species co-occurrence patterns to match chance expectations if stochastic factors dominate (Gotelli \& McCabe, 2002; Veech, 2014).

Examining overall patterns of co-occurrence across a community matrix can reliably detect non-random patterns. However, because overall C-scores lump together aggregated and segregated species pairs, they fail to distinguish the directions of deviation when using with fixed-fixed null models (Gotelli \& Ulrich, 2012; Ulrich \& Gotelli, 2013). Further ambiguity emerges from the fact that multiple processes contribute to the observed patterns. For example, although segregation is often attributed to competition (Diamond, 1975), it can also result from habitat affinities for non-overlapping habitats, dispersal limitation or historical effects (Gotelli \& McCabe, 2002). Similarly, the same process can also generate different patterns.
For example, 10 species might exist in two groups where each species in the first group competitively excludes exactly one from the second group while not affecting other species within that or its own group. This would generate strongly segregated and aggregated species pairs within the community matrix from the same mechanism. Thus, matrix-wide average co-occurrence statistics cannot disentangle the direction of deviation and mechanisms of community assembly.

To resolve these ambiguities, researchers have begun instead to analyse associations between particular pairs of species (Sfenthourakis et al., 2006; Gotelli \& Ulrich, 2010; Veech, 2013, 2014; Blois et al., 2014). A recent promising approach uses the geographical distances among sites and data on their differences in environmental conditions to infer the likely relative importance of environmental filtering, biotic interactions and dispersal limitation (Blois et al., 2014). If dispersal limitation is important, we expect the extent of segregation between species pairs to increase with increasing geographical distance. If environmental filtering is important, we expect degrees of segregation between species pairs to increase as the environmental distance between their sites increases. Such approaches are particularly useful for comparative studies where communities with different dynamics are being compared or one community is being tracked through time in relation to changing circumstances. Although the analysis of associations between particular pairs of species still faces problems like non-independence in the occurrences of species pairs, it offers ecologists considerably more potential for inferring processes from patterns (Blois et al., 2014; Veech, 2014). Although conclusions from correlative studies will always be contingent, using data from many species distributed over many sites with contrasting site conditions will tend to make inferences more reliable.

As environmental conditions and resource availability change, we expect biotic interactions and patterns of species co-occurrence to also change as species go extinct, ranges shift and patterns of relative abundance change (Blois et al., 2013). This motivates us to move beyond inferring static patterns of species co-occurrence to explore how these shift over time, and what this tells us about changes in the mechanisms driving community assembly. High-quality long-term data sets, however, are scare, limiting our ability to analyse such dynamics (but see Rooney, 2008).

Here, we use resurvey data from the same set of sites to infer and compare the relative importance of the mechanisms driving community assembly in three distinct Wisconsin forest plant communities. We then assess how these mechanisms have changed over the past 50 years. We specifically ask:

1. Are overall (community matrix-wide) patterns of plant co-occurrence mostly random within these communities? Have these patterns become more or less random since the 1950s?

2. What patterns of co-occurrence emerge when we analyse associations between particular pairs of species? Can we use these pair-wise results to infer the relative importance of habitat filtering, dispersal limitation and species interactions in structuring these plant communities? How do these differ among the communities? Have they changed over time? 
3. To what degree do these mechanisms tend to generate aggregated and segregated species pairs? Do both kinds of association contribute similarly to our ability to infer the mechanisms operating in these communities?

Our approach is based on observed patterns in natural communities rather than manipulative experiments and thus suggests the mechanisms likely to be operating rather than pinpointing their exact relevance. This limits the ability to discriminate finely among the alternative mechanisms that may operate in combination to structure plant communities. Nevertheless, with sufficient data, the methods used here can identify the mechanisms likely to be acting and their relative importance. We therefore present our results to capitalize on extant field data and address one of the most fundamental questions in ecology. Therefore our focus here is more on generating than testing hypotheses.

\section{METHODS}

Figure 1 shows a flowchart of the analyses conducted in this study.

\section{Vegetation data}

The diligent efforts of J. T. Curtis and his students generated detailed plant community data for more than a thousand sites across Wisconsin from the 1950s (Curtis, 1959). Data from these sites provide valuable baselines for inferring long-term ecological change when combined with subsequent resurveys of the same sites in the 2000s (Waller et al., 2012), Here, we take advantage of the number and quality of these data to analyze patterns of species co-occurrence among 266 forested sites distributed among three community types: 108 northern upland forest (NUF) stands, 30 stands in the central sand plain pine barrens (CSP) and 128 southern upland forests (SUF) stands (Fig. 2).

At each site, researchers estimated the frequency of all vascular plants present at the site by recording their presence across many spaced $1 \mathrm{~m} \times 1 \mathrm{~m}$ quadrats (see Waller et al., 2012, for sampling details). In the 1950 s, researchers usually sampled 20 $1 \mathrm{~m} \times 1 \mathrm{~m}$ quadrats per site. Sampling efforts increased considerably in the 2000s. However, because sampling effort can affect
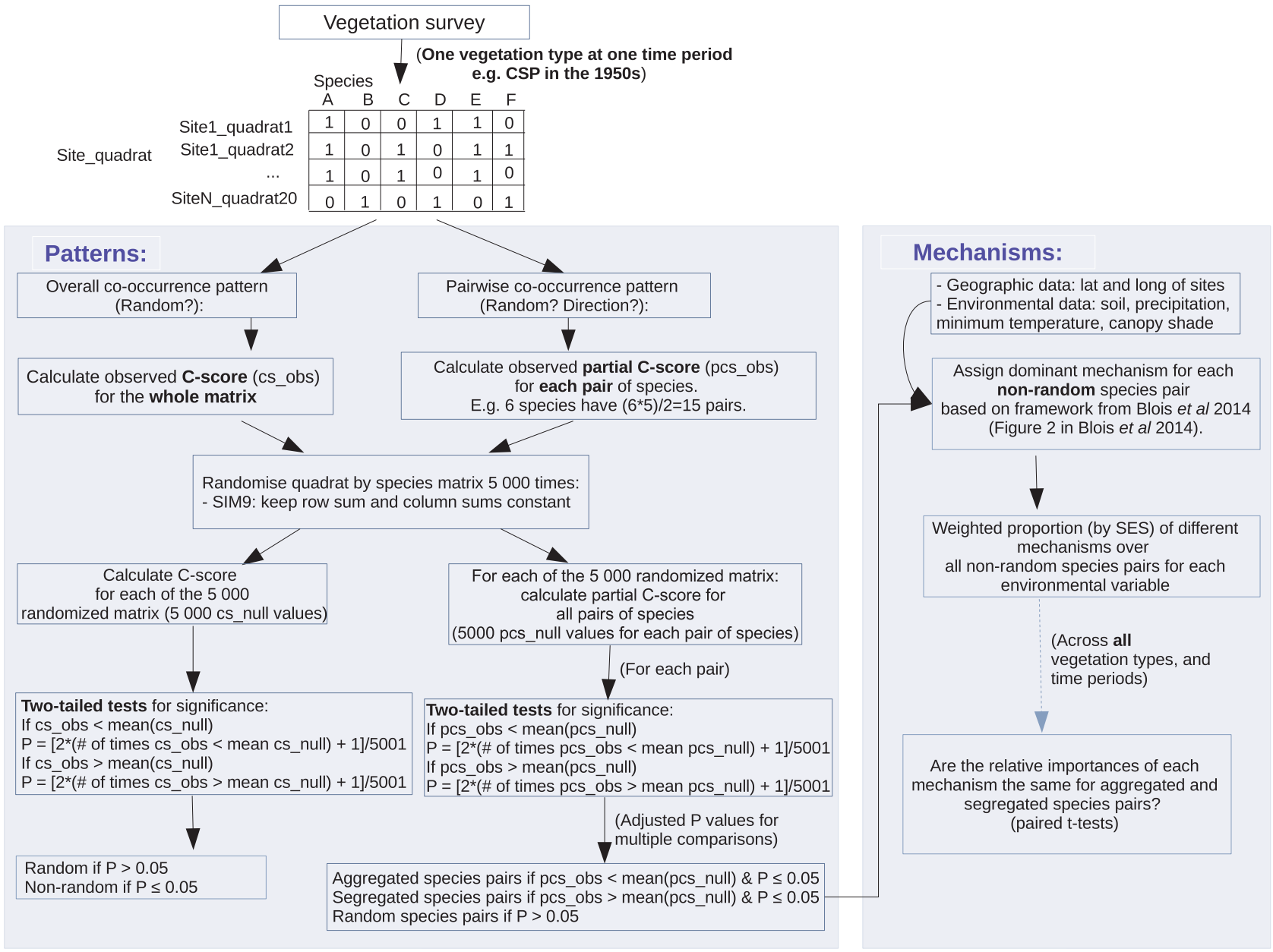

Figure 1 Flowchart of analyses conducted in this study. 
Plant co-occurrence dynamics in Wisconsin

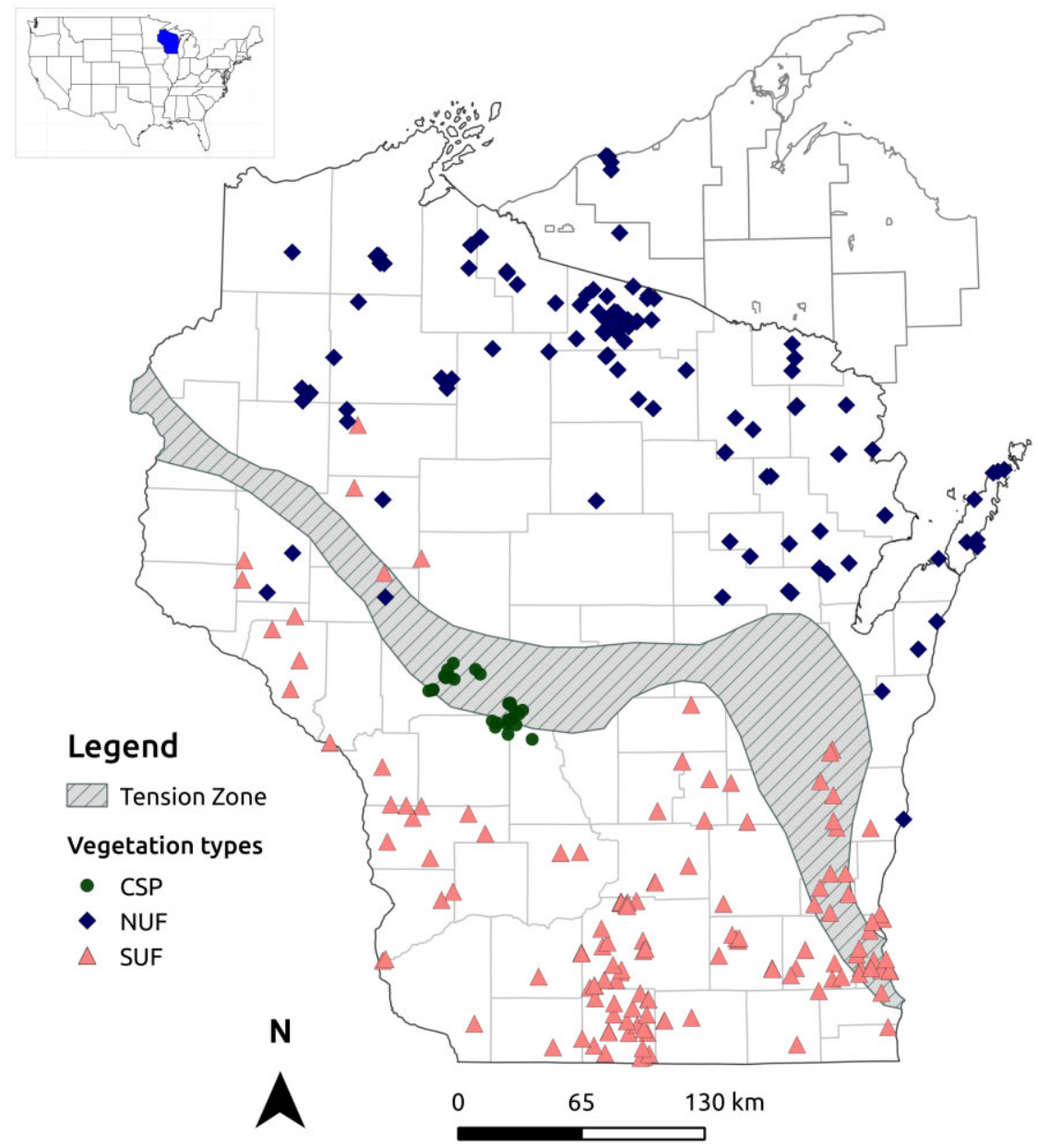

Figure 2 Locations of forest stands sampled in both the 1950s and the 2000s. The tension zone in the map corresponds to sharp gradients in temperature, precipitation and snow depth. Symbols indicate three general vegetation types: northern upland forest (NUF), central sand plain pine barrens (CSP) and southern upland forest (SUF). co-occurrence patterns (Horner-Devine et al., 2007; Gotelli \& Ulrich, 2010; Pitta et al., 2012), we evenly sub-sampled 20 quadrats from the more extensive 2000s data to equalize sampling efforts across time periods.

Within each vegetation type and time period, we stacked the quadrat by species matrices for all sites into a single matrix where each row $r_{\mathrm{Q} q}$ represents quadrat $q$ within site $Q$, and each column represents a species (Fig. 1). Each cell in the matrix contains either 1 or 0 , indicating the presence or absence of species in quadrat $q$ of site $Q$, respectively. Thus, the row sum represents the number of species (species richness) in that quadrat of a given site while the column sum represents the number of occurrences of that species across all quadrats and sites. We only include species observed in more than five quadrats to exclude rare species and facilitate our ability to determine core significant co-occurring species pairs. We investigate overall and pair-wise co-occurrence patterns using these six matrices (three vegetation types $\times$ two time periods). We consider the $1-\mathrm{m}^{2}$ quadrat as our sample unit for analysing patterns of species co-occurrence. Species that co-occur on this scale are more likely to directly interact than scoring co-occurrence across the much larger sampled area at each site $\left(>2000 \mathrm{~m}^{2}\right)$.

\section{Environmental data}

We characterized the environmental conditions present at each site using data on both climate and soil conditions. For climate, we extracted daily precipitation and minimum temperature of each site between 1950 and 2006 from a database provided by Kucharik et al. (2010). For sites in the NUF and SUF (originally sampled between 1948 and 1955), we used the mean climate data from 1950 to 1956 as a proxy for 1950s climate and data from 2000 to 2006 as a proxy for the 2000s. For sites in the CSP (originally sampled in 1958), we used mean climate data from 1955 to 1959 and from 2002 to 2006. Soil properties were measured for all sites, including soil $\mathrm{pH}$, organic matter content, soil texture, nitrogen, phosphorus, potassium, calcium and magnesium content. These data were also used for the 1950s as soil data were not available for that time period. Principal component analyses (PCA) of the soil variables across sites within each vegetation type and time period yielded first axes that all explained over $70 \%$ of the total variance. We therefore used scores on these first axes to represent soil conditions at each site. Because the CSP sites changed conspicuously in canopy coverage between the 1950s and 2000s (Li \& Waller, 2015), we include canopy coverage as an additional environmental variable. In 
summary, we used precipitation, minimum temperature, soil PCA axis 1 (soil hereafter) and canopy coverage for the CSP sites and the same environmental variables except canopy coverage for the SUF and NUF sites.

\section{Null models and the randomization algorithm}

Two null models are commonly used in species co-occurrence studies: null models that maintain species occurrence totals and species richness at sample units (SIM9; Gotelli, 2000) and null models that maintain species occurrence totals but allow species richness at sample units to vary (SIM2; Gotelli, 2000). The object in using null models is to only vary the testing variable of interest (species associations here) while keeping all other things unchanged (Gotelli, 1996). As a result, Gotelli (2000) argues that SIM9 is most appropriate for 'island lists' type samples where sites differ in species composition or habitats while SIM2 is most appropriate for 'sample lists' where samples have been collected in areas of homogeneous habitat. As our samples come from well-separated sites distributed across a large region that differ considerably in environmental conditions (see Fig. A1 in Appendix S1 in the Supporting Information) and species composition (Fig. A2 in Appendix S1), it is more reasonable to treat them as islands rather than samples within homogeneous habitat. We therefore apply the more stringent SIM9 null model that fixes both species richness and species occurrences (row and column totals). This preserves all variation present in species occurrences and the number of species present within quadrats. SIM9 generates null models that benchmark tests show to have high power and low probabilities of Type I and Type II errors (Gotelli, 2000; Gotelli \& Ulrich, 2010).

To assess overall and pair-wise species co-occurrence, we randomized species occurrences within vegetation types and time periods 5000 times using the SIM9 null model. We used a 'trial-swap' method (Miklós \& Podani, 2004), a modification of the sequential swap method (Gotelli, 2000) with better performance. SIM9 has been used in most species co-occurrence studies (e.g. Gotelli \& McCabe, 2002; Pitta et al., 2012; Larsen \& Ormerod, 2014), but SIM2 is not rare in the co-occurrence literature (e.g. Krasnov et al., 2014). We also report results from SIM2 in Appendix S2 for comparison with the results from SIM9 and to allow comparison with other studies using SIM2. These SIM2 results support most of our findings based on SIM9, though they differ in detail. We do not discuss the SIM2 results further here.

\section{Overall co-occurrence patterns}

For each vegetation type (NUF, SUF, CSP) and each time period (1950s, 2000s), we quantified overall species co-occurrence patterns using the matrix-wide method. This approach tests the null hypothesis that the community is unstructured in the sense that species co-occur randomly. It calculates C-scores based on quadrats by species matrix (see Fig. 1). C-scores are computed from the mean number of checkerboard units observed among all possible species pairs within a matrix with
C-score $=\frac{\sum_{i=1}^{s} \sum_{j=1}^{i-1}\left(c_{i}-m_{i j}\right)\left(c_{j}-m_{i j}\right)}{s(s-1) / 2}$,

where $s$ is the number of species, $c_{i}$ and $c_{j}$ are the number of quadrat occurrences of species $i$ and $j$ and $m_{i j}$ is the number of quadrats where both species occurred. To assess whether the observed co-occurrence pattern was random, we compared the observed C-score with a null distribution of C-scores generated from the 5000 null matrices. From this, we calculated a $P$-value from the rank of the observed C-score using a two-tailed test for each matrix. If $P<0.05$, we rated the overall co-occurrence pattern in that vegetation type and time period as non-random. To compare the deviations from random expectation over time, we also calculated the standardized effect size (SES): $\mathrm{SES}=\left(X_{\mathrm{obs}}-\bar{X}_{\text {rand }}\right) / \mathrm{SD}_{\text {rand }}$ where $X_{\mathrm{obs}}$ is the observed C-score and $\bar{X}_{\text {rand }}$ and $\mathrm{SD}_{\text {rand }}$ are the mean and standard deviation of the C-scores generated by the simulations. We use the pair of SES values for each vegetation type to compare the changes across time periods in overall co-occurrence.

\section{Pair-wise co-occurrence patterns}

To determine non-random species pairs (aggregated or segregated), we calculated pair-wise association values using the 'partial C-score' (the number of checkerboard units of a given species pair). For each species pair, the partial C-score can be calculated as $\left(c_{i}-m_{i j}\right)\left(c_{j}-m_{i j}\right)$, where $c_{i}, c_{j}$ and $m_{i j}$ are defined above. To determine whether a pair of species co-occurred more or less than expected by chance, we compare the observed partial C-score for each species pair against expected values calculated from the 5000 simulated matrices generated in the SIM9 procedure. For each species pair, a $P$-value was calculated based on the rank of observed partial C-score using a two-tailed test. To reduce false positives, we adjusted $P$-values by controlling for the false discovery rate within each vegetation matrix (Benjamini \& Hochberg, 1995). We also again calculated SES for each species pair as $\mathrm{SES}=\left(X_{\mathrm{obs}}-\bar{X}_{\text {rand }}\right) / \mathrm{SD}_{\text {rand }}$ where $X_{\mathrm{obs}}$ is the observed partial C-score and $\bar{X}_{\text {rand }}$ and $\mathrm{SD}_{\text {rand }}$ are the mean and standard deviation of the partial C-scores in simulations. If the adjusted $P$-value is $<0.05$ for a given species pair, we concluded that the co-occurrence pattern for that species pair was non-random. Non-random species pairs with $\mathrm{SES}<0$ reflect positive co-occurrence (aggregation) as they co-occur more than expected by chance. Non-random species pairs with SES $>0$ reflect negative co-occurrence (segregation) as they co-occur less often than expected by chance. Within each vegetation type, we then tested whether the pair-wise co-occurrence pattern changed over time, using chi-square tests on a $2 \times 2$ contingency table. The contingency table for each vegetation type records the number of random or non-random (aggregated and segregated) species pairs in the 1950s and 2000s.

\section{Mechanisms driving co-occurrence patterns}

For each non-random species pair ( $a$ and $b$ ) in a given vegetation type and time period, sites can be classified into one of four 
mutually exclusive groups: species $a$ only $(1,0)$, species $b$ only $(0,1)$, both species $a$ and $b$ present $(1,1)$, both species $a$ and $b$ absent (0,0) (Blois et al., 2014). The environmental characteristics and locations of these groups of sites are then be used to infer probable causes of non-randomness of that species pair. We inferred significant species pairs from the quadrat data yet we only have environmental data for whole sites. We therefore compared geographical distances and environmental distances among sites rather than quadrats to test non-random species pairs.

We used analyses of variance to test the significance of differences in geographical distances and environmental distances between groups of sites, applying these tests to $(1,1)$ and $(0,0)$ sites for the aggregated pairs and $(1,0)$ or $(0,1)$ sites for the segregated pairs. We only used species pairs with more than five $(1,1)$ and $(0,0)$ sites for aggregated pairs and more than five $(1,0)$ and $(0,1)$ sites for segregated pairs to ensure meaningful results. To compare geographical distances, we used a one-way permutational multivariate analysis of variance (PMANOVA) to compare the latitude and longitude between these site groups. To test whether these groups of sites differed in environmental conditions, we applied one-way analyses of variance (ANOVA) to each environmental variable [i.e. soil, precipitation, minimum temperature, and shade (for the CSP sites)]. We did not report $P$-values from these tests as we used them only to assign a dominant underlying mechanism to each non-random species pair. We did not impose a false positive correction on these PMANOVA and ANOVA results as that would result in over-conservative tests (Blois et al., 2014).

Following Blois et al. (2014), we then used results from these PMANOVA and ANOVA analyses to infer probable dominant mechanisms for each species pair (Fig. A3 in Appendix S1). If geographical and environmental distances between these two groups of sites differ significantly, dispersal limitation and/or environmental filtering is important. If geographical distances differ significantly, but not environmental distances, dispersal limitation is likely to play an important role. If environmental distances differ significantly but not geographical distances, it is likely that environmental filtering is occurring. Finally, if neither geographical distances nor environmental distances differ significantly, we infer that species interactions are probably important.

This approach allowed us to assign a likely dominant mechanism to each species pair that showed a significant association. These analyses also yielded SESs for the each of these partial C-scores. This allowed us to weight each of the assigned mechanisms by the absolute values of these SESs. Using this procedure has the effect of ensuring that the mechanisms assigned reflect our level of statistical confidence in each assignment. We then calculated the weighted relative proportion of each mechanism for each combination of environmental variable, vegetation type and time period. For example, for the SUF at the 2000s, we calculated the weighted relative proportion of each mechanism for soil, precipitation and minimum temperature. We then compared these weighted relative proportions qualitatively among these vegetation types and between time periods.
We also tested whether aggregated and segregated species pairs suggest similar or different underlying processes. To do this, we applied a paired $t$-test to compare the weighted proportions of each mechanism between the aggregated and segregated species pairs as estimated for each environmental variable, vegetation type and time period. For each mechanism, this yielded a sample size of 20 based on the four environmental variables in the CSP $(\times$ two time periods $=8)$ plus the three environmental variables in the SUF and NUF $(\times$ two time periods $=6+6=12$; see Fig. A4 in Appendix S1). If all mechanisms show a similar relative importance for the aggregated and segregated species pairs, aggregation and segregation may be shaped by the same processes.

All analyses were conducted using R v.3.1.0 (R Core Team, 2015), with the 'vegan' package (Oksanen et al., 2013) for the null models.

\section{RESULTS}

\section{Overall co-occurrence patterns}

Overall patterns of association in all three vegetation types were non-random as indicated by the C-scores in both the 1950s and 2000s (all $P<0.001 ;$ Table 1). Average overall levels of co-occurrence remained stable in the NUF and SUF, but not the CSP (SES of C-scores declined 35\%), between the 1950s and 2000s.

\section{Pair-wise co-occurrence patterns}

After excluding species that occurred in fewer than five quadrats and controlling for false discovery rates, $8.7-14 \%$ of the species pairs were significantly aggregated and $7.5-17.5 \%$ of the pairs were segregated (Table 2 ). Slightly more species pairs were segregated than aggregated across the broad regions of the SUF and NUF but the geographically restricted CSP communities

Table 1 Community-wide co-occurrence.

\begin{tabular}{lcllll}
\hline $\begin{array}{l}\text { Vegetation } \\
\text { type }\end{array}$ & Date & $\begin{array}{l}\text { Species } \\
\text { richness }\end{array}$ & $\begin{array}{l}\text { Species } \\
\text { used }\end{array}$ & SES & $P$-value \\
\hline NUF & $1950 \mathrm{~s}$ & 245 & 146 & 2.67 & $<0.001$ \\
NUF & $2000 \mathrm{~s}$ & 255 & 160 & 2.83 & $<0.001$ \\
CSP & $1950 \mathrm{~s}$ & 105 & 61 & 6.86 & $<0.001$ \\
CSP & $2000 \mathrm{~s}$ & 136 & 55 & 4.46 & $<0.001$ \\
SUF & $1950 \mathrm{~s}$ & 365 & 225 & 2.08 & $<0.001$ \\
SUF & $2000 \mathrm{~s}$ & 324 & 186 & 2.00 & $<0.001$ \\
\hline
\end{tabular}

NUF, northern upland forest; CSP, central sand plain pine barrens; SUF, southern upland forest; SES, standardized effect size.

Overall levels of departure from random patterns of species co-occurrence for each of the three forest types and both time periods. The SES and $P$-values refer to how far the observed checkerboard score departs from the corresponding null distribution. The 'Species used' column represents the number of species that were observed in more than five quadrats at each vegetation type and time period. 
Table 2 Pair-wise species associations.

\begin{tabular}{|c|c|c|c|c|c|}
\hline $\begin{array}{l}\text { Vegetation } \\
\text { type }\end{array}$ & Date & Total pairs & Random pairs & $\begin{array}{l}\text { Aggregated pairs } \\
(P<0.05)\end{array}$ & $\begin{array}{l}\text { Segregated pairs } \\
(P<0.05)\end{array}$ \\
\hline NUF & $1950 \mathrm{~s}$ & 10,585 & $7,319(69.1 \%)$ & $1,480(14.0 \%)$ & $1,786(16.9 \%)$ \\
\hline NUF & $2000 \mathrm{~s}$ & 12,720 & $8,867(69.7 \%)$ & $1,630(12.8 \%)$ & $2,223(17.5 \%)$ \\
\hline CSP & $1950 \mathrm{~s}$ & 1,830 & $1,402(76.6 \%)$ & $238(13.0 \%)$ & $190(10.4 \%)$ \\
\hline CSP & $2000 \mathrm{~s}$ & 1,485 & $1,244(83.8 \%)$ & $129(8.7 \%)$ & $112(7.5 \%)$ \\
\hline SUF & $1950 \mathrm{~s}$ & 25,200 & $18,793(74.6 \%)$ & $2,734(10.8 \%)$ & $3,673(14.6 \%)$ \\
\hline SUF & $2000 \mathrm{~s}$ & 17,205 & $13,553(78.8 \%)$ & $1,728(10.0 \%)$ & $1,924(11.2 \%)$ \\
\hline
\end{tabular}

Columns show the total number of pairs of species analysed and the numbers (and percentage) of those pairs that displayed random, aggregated or segregated patterns of association in each forest type and period based on statistical tests of the observed checkerboard scores using null models, corrected for the false discovery rate. Abbreviations are as in Table 1.

Table 3 Relative importance of inferred mechanisms.

\begin{tabular}{lllrl}
\hline Mechanisms & $\begin{array}{l}\text { Aggregated } \\
(\text { mean } \pm \text { SE) }\end{array}$ & $\begin{array}{l}\text { Segregated } \\
(\text { mean } \pm \text { SE) }\end{array}$ & \multicolumn{1}{c}{$t$} & $P$-value \\
\hline Dispersal limitation & $0.207(0.033)$ & $0.181(0.031)$ & 1.668 & 0.11 \\
Environmental filtering & $0.197(0.029)$ & $0.212(0.035)$ & -0.657 & 0.52 \\
Dispersal limitation/filtering & $0.165(0.025)$ & $0.176(0.031)$ & -1.192 & 0.25 \\
Species interactions & $0.432(0.044)$ & $0.430(0.043)$ & 0.081 & 0.94 \\
\hline
\end{tabular}

The frequencies (values sum to 1 ) at which the various potential underlying mechanisms were inferred from the patterns of aggregated and segregated species co-occurrences, averaged across all environmental variables, vegetation types and time periods. The final columns show paired $t$-statistics and associated $P$-values for testing the hypothesis that the weighted relative importance is the same between aggregated and segregated species pairs. Note the match in these frequencies across all inferred mechanisms.

showed the opposite trend (Table 2). The proportion of significantly associated species pairs (aggregated and segregated) has remained constant over the past half century across the more continuous forests of northern Wisconsin, but associations have decreased in the more fragmented forests of the CSP and SUF $\left(P<0.001\right.$, with $\chi_{1}^{2}$ of 25.6 and 99.36 , respectively).

\section{Mechanisms driving co-occurrence patterns}

Environmental filtering, biotic interactions and dispersal limitation all appear to have contributed to these patterns of species association, but their relative importance varies among the three communities (Fig. 3). Species interactions appear to have been a dominant force structuring the CSP communities in the 1950s but their contributions have declined somewhat since then. Nevertheless, these still remain stronger in the CSP than in the other two community types, perhaps reflecting a larger role for environmental differences in those other, more heterogeneous, communities. Dispersal limitation is inferred to have affected the structure of the fragmented SUF considerably more than the other community types. In addition, its relative importance has increased over the past 50-60 years in both the SUF and CSP communities. In contrast, environmental filtering appears to play a stronger role in the NUF than in the SUF region. Shade (canopy coverage) within the CSP communities also appears to have been an important environmental factor structuring those communities in the 1950s but its influence appears to have declined as these forests have filled in and increased in basal area. The relative importance of all mechanisms appears to have been most stable in the NUF communities. This stability may reflect the fact that northern Wisconsin has retained most of its forest cover and experienced less ecological change than other regions of the state.

The relative importance of these mechanisms did not differ between aggregated and segregated pairs across all environmental variables, vegetation types and time periods (all $P>0.1$; Table 3). This suggests that aggregated and segregated species pairs can be shaped by the same ecological processes.

\section{DISCUSSION}

Our study differs from most previous studies of species co-occurrence in using a finer geographical scale, involving many sites and in using both current and historical data. We analysed patterns at hundreds of sites distributed among three community types using both legacy data from the 1950s and modern resurvey data at the same sites from the 2000s. This amount and quality of data gave us considerable power to detect patterns, to characterize the potential mechanisms 

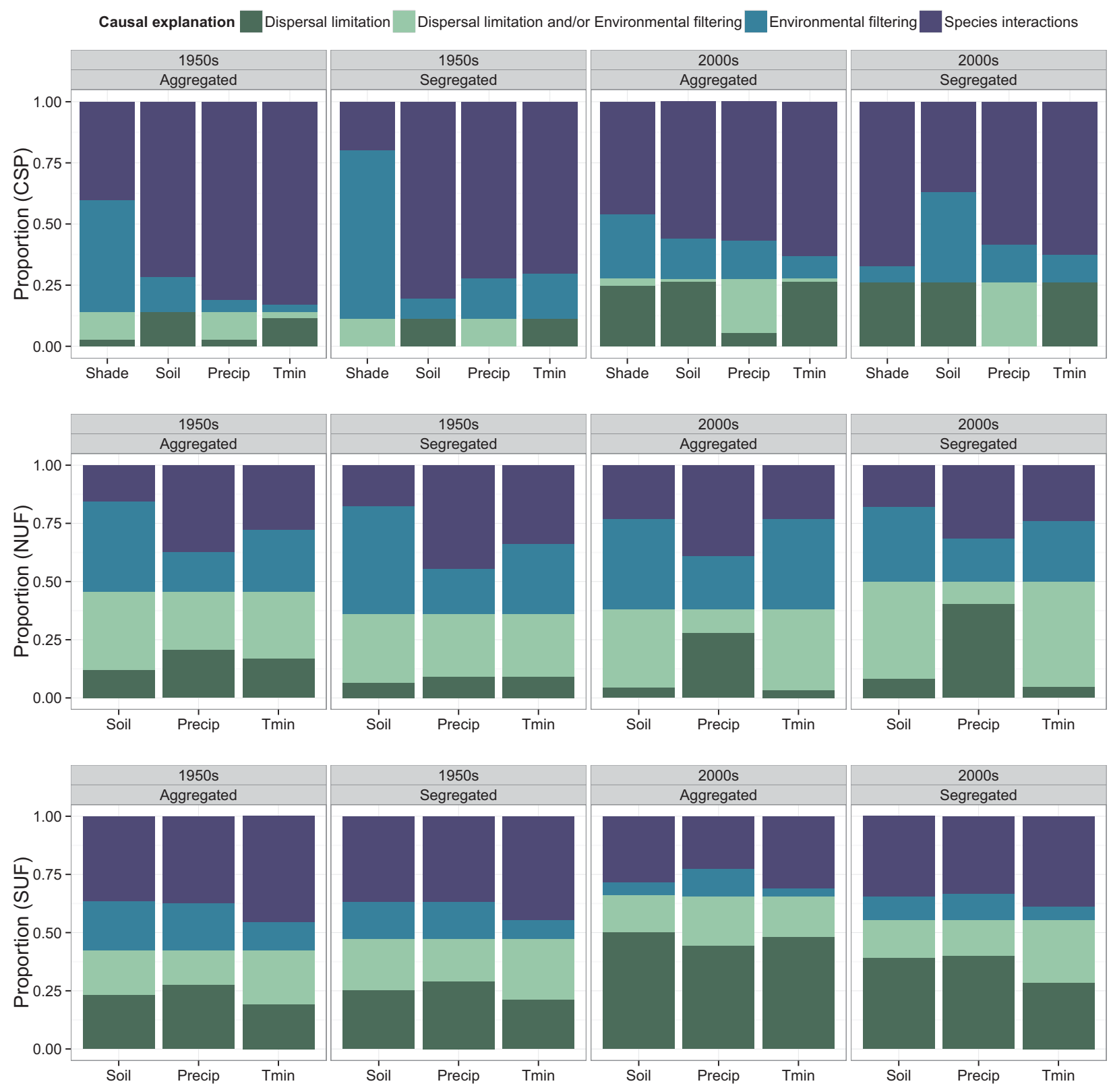

Figure 3 Comparison of community assembly mechanisms (environmental filtering, dispersal limitation, dispersal limitation and/or environmental filtering, and species interactions) among the three forest community types and over time (abbreviations are as in Fig. 2). Each bar shows the relative importance of mechanisms contributing to aggregated/segregated species pairs in each forest type and period as inferred using one of the following environmental variables: Soil, precipitation (Precip), minimum temperature (Tmin), and canopy cover (Shade). Note the different profiles across forest types, the consistency of mechanisms in the NUF, the increased roles of dispersal limitation in the CSP and SUF communities in the 2000s and the similar proportions of mechanisms for segregated and aggregated species pairs.

responsible for those patterns, to compare these across community types and to track changes in their relative importance over time.

\section{Co-occurrence patterns}

Overall patterns of plant co-occurrence were non-random across all three forest types in both time periods with more segregation than expected by chance (i.e. more checkerboard pairs) in all matrices based on SESs of C-scores (Table 1). These results resemble those from similar empirical studies (Gotelli \& McCabe, 2002; Pitta et al., 2012). The overall strength of these co-occurrence patterns (as judged by the SES scores) declined over the last 50 years in forests of the CSP but remained stable in the SUF and NUF of northern Wisconsin (as noted in a previous study of the NUF; see Rooney, 2008). 
In our study, after controlling for false positive discovery rates, we observed $16-31 \%$ non-random pairs (Table 2). These proportions exceed those observed in most similar studies. This may reflect our large sample sizes and fine taxonomic and spatial resolution. Using the same framework and Quaternary fossil pollen data, Blois et al. (2014) found $2 \%$ at most of plant species pairs to be significant. However, their data only extend to the genus level, omitting many species, and span a continental scale. Using the same null model, Pitta et al. (2012) found fewer than $10 \%$ of species pairs in their plant communities to have significant associations, perhaps reflecting their coarser geographical scale. In contrast, our analyses are based on $1-\mathrm{m}^{2}$ quadrats, within which microenvironmental conditions vary little, making aggregation and segregation more likely.

Both the average overall and species pair co-occurrence patterns in the NUF have remained stable over the past half century. In the CSP, however, the proportions of aggregated and segregated pairs have declined, as have segregated pairs in the SUF communities (Table 2). These communities have also suffered habitat fragmentation and pronounced biotic homogenization (Rogers et al., 2008; Li \& Waller, 2015) which could play a role. As community composition converges among sites, the proportion of significantly associated species pairs may decrease as the same reduced subset of common species come to dominate most sites (Olden \& Poff, 2003). We also see an increase in the role played by stochastic forces such as dispersal limitation (Fig. 3). This could also limit the intensity of competitive interactions and corresponding selection pressures. Dispersal barriers from habitat isolation and/or declines in seed dispersers probably decrease the number of sites at which two species can co-occur. Thus, habitat fragmentation can limit interactions among species. Such declines in these interactions might also eventually threaten ecosystem functions and services (Díaz et al., 2013) and the ability of these communities to respond to environmental changes (Suttle et al., 2007).

\section{Mechanistic processes}

Observing fewer co-occurrences than expected by chance has long been interpreted as a signature of competition (Diamond, 1975). However, most such assessments were based only on community-wide patterns of association that confound positive and negative associations, ignore finer-scale interactions and do not evaluate relationships to geographical or environmental distance to discriminate among the alternative mechanisms that could drive such associations. We have known for some time that differential habitat affinities, differences in dispersal ability and historical factors such as allopatric speciation can all also produce community-wide associations (Gotelli \& McCabe, 2002; Sfenthourakis et al., 2006).

The methods we explored here use instead associations between particular pairs of species assessed on a fine $\left(1-\mathrm{m}^{2}\right)$ scale. When coupled with extensive vegetation and environmental data, these give us the potential to identify the mechanisms contributing to community assembly including limited dispersal, species interactions and adaptations to particular soil or other environmental conditions (Blois et al., 2014; Veech, 2014). We should, of course, apply these methods with caution and keep their assumptions and limitations in mind. Specifically, we assumed that species pairs with significant associations that did not decline with geographical distance or differences in environmental conditions probably reflect species interactions. However, other unmeasured environmental variables could also have contributed to these associations (Blois et al., 2014). In addition, results generated by the framework of Blois et al. (2014) depend on the environmental variables measured and the existence of an adequate number of significant associations. This suggests that this approach is best suited for use in comparative studies that analyse community co-occurrences in detail using standardized sampling across a large spatial and temporal scales. In addition to interpreting results carefully, it would be ideal to supplement them with experimental work. Limitations clearly exist here. Performing the experiments necessary to confirm the relative importance of these mechanisms in three current communities would entail a great amount of effort and take years. In addition, we cannot go back in time to perform such experiments on the communities of 50 years ago. Given these limitations, we urge ecologists to use the data and methods available to make more inferences and comparisons like those presented here while testing these, where possible, with experiments and simulations.

Environmental filtering, species interactions and dispersal limitation all appear to play roles in structuring these forest communities. However, their relative importance appears to vary both among the three community types and over time (Fig. 3). The particular environmental variable examined also influenced the relative importance of the mechanisms inferred to be important. For example, in the CSP at the 1950s, environmental filtering dominated when tested with canopy shade but not with other variables (Fig. 3). This result demonstrates the importance of testing several environmental variables when using the framework of Blois et al. (2014) to assess the relative roles of alternative processes. Species interactions appeared to play a bigger role in structuring the CSP communities in both periods but were less important in the SUF and NUF of Wisconsin. This may reflect the fact that the CSP community type is restricted in geographical range and the range of environments it occupies, limiting the role of environmental filtering in these stands.

We randomized within each vegetation type and across all sites rather than testing for associations within individual sites. This scale of analysis may tend to emphasize environmental filtering more than species interactions because environmental filtering often acts at a coarser scale than species interactions. Nevertheless, we still found species interactions to be more important than environmental filtering in most cases (Fig. 3). This suggests that the scale at which we analysed these data did not bias our results.

The mechanisms inferred in this study provide ecological insights that match our knowledge about these communities. Our analyses of the 1950s CSP communities suggest that canopy openness and species interactions greatly affected community 
assembly there. Previous work confirms that fire maintained these pine barren communities before the 1950s (Habeck, 1959). Fire suppression subsequently allowed these communities to succeed into closed canopy forests initiating a contrasting set of processes ( $\mathrm{Li} \&$ Waller, 2015). These CSP sites are all physically close to one another and located on similarly sandy soils, limiting the extent to which differences in soil or climate can contribute to among-site differences via environmental filtering. This limitation is evident in the upper panel of Fig. 3. Soil and climate appear to have stronger effects in the NUF compared with the SUF. The NUF and SUF are separated by a northwest-southeast tension zone (Fig. 2) that corresponds to sharp gradients in temperature, precipitation and snow depth (Curtis, 1959). Sites north of the tension zone have higher precipitation and evapotranspiration ratios, cooler temperatures and more leaching than those south of the tension zone, making soils in the NUF sites poorer than those in the SUF. Climate conditions are also more severe in the NUF. These facts may explain why environmental filtering by soil and climate is stronger in the NUF than the SUF.

Changes in the importance of the mechanisms also matched changes we observed in the patterns of species associations. For example, the proportions of non-random species pairs decreased in the CSP and SUF where the inferred relative importance of species interactions decreased (Fig. 3). This may reflect increases in the stochastic forces now influencing community structure in these increasingly fragmented habitats. For the NUF, both co-occurrence patterns and the relative importance of different mechanisms remained stable over time.

We were somewhat surprised to find that the relative importance of each mechanism for aggregated and segregated species pairs was similar across all environmental variables, vegetation types and time periods. This suggests that both segregated and aggregated species pairs may result from the same community assembly processes. This seems counter-intuitive, as aggregation and segregation are opposing results. However, similar processes can produce such results, as illustrated by the example of competitive exclusion in the Introduction. Similarly, for species to survive at a given site they need to pass a series of 'filters' (e.g. dispersal barriers, environmental limitations and biotic interactions including herbivory and competition). Species in aggregated pairs both have to pass these filters while species in segregated pairs reflect situations where one or both did not pass at least one of these filters. Thus the same 'filtering' process can produce both patterns of association. An analogous example is when local observed species diversity is compared with the 'dark' diversity of species not observed but theoretically able to inhabit the site (Pärtel et al., 2011). Again, similar community assembly processes yield different patterns.

\section{CONCLUSIONS}

The same mechanisms appear to drive the formation of both segregated and aggregated species pairs here, a novel finding that deserves to be tested more widely. We found strong differences in the mechanisms structuring different types of forest commu- nities. These differences reflect known or suspected differences among them in environmental conditions and dynamics. Despite apparent stasis in the forces governing community structure in the more intact and recovering northern Wisconsin forests, we observed marked increases in inferred levels of dispersal limitation over the last 50 years in the fragmented landscapes of southern Wisconsin. These forests are also paying an extinction debt and experiencing biological homogenization (Rogers et al., 2008, 2009; Li \& Waller, 2015) as well as the declines in associations documented here. Together, these trends suggest that stochastic forces are now playing stronger roles in these communities. This leads us to the conjecture that fragmented forests generally are becoming more susceptible to dispersal limitation and 'ecological drift'.

\section{ACKNOWLEDGEMENTS}

We are grateful that J. T. Curtis and his students collected and archived baseline data in the 1950s. We also thank previous researchers in the Waller Lab, including but not limited to $\mathrm{T}$. Rooney, S. Wiegmann and D. Rogers, for their resurvey work in the 2000s. This study would have been impossible without these baseline and resurvey data. Comments by Alison Paulson, editor David Currie and Richard Field, and two anonymous referees greatly improved the paper. Financial support was provided by the National Science Foundation Dimensions of Biodiversity program via grant DEB 1046355.

\section{REFERENCES}

Benjamini, Y. \& Hochberg, Y. (1995) Controlling the false discovery rate: a practical and powerful approach to multiple testing. Journal of the Royal Statistical Society: Series B (Statistical Methodology), 57, 289-300.

Blois, J.L., Zarnetske, P.L., Fitzpatrick, M.C. \& Finnegan, S. (2013) Climate change and the past, present, and future of biotic interactions. Science, 341, 499-504.

Blois, J.L., Gotelli, N.J., Behrensmeyer, A.K. et al. (2014) A framework for evaluating the influence of climate, dispersal limitation, and biotic interactions using fossil pollen associations across the late Quaternary. Ecography, 37, 1095-1108.

Chase, J.M. \& Leibold, M.A. (2003) Ecological niches: linking classical and contemporary approaches. University of Chicago Press, Chicago, IL.

Curtis, J.T. (1959) The vegetation of Wisconsin. University of Wisconsin Press, Madison, WI.

Díaz, S., Purvis, A., Cornelissen, J.H.C. et al. (2013) Functional traits, the phylogeny of function, and ecosystem service vulnerability. Ecology and Evolution, 3, 2958-2975.

Diamond, J.M. (1975) Assembly of species communities. Ecology and evolution of communities (ed. by M.L. Cody and J.M. Diamond), pp. 342-444. Harvard University Press, Cambridge, MA.

Gotelli, N.J. (1996) Null models in ecology. Smithsonian Institution Press, Washington, DC. 
Gotelli, N.J. (2000) Null model analysis of species co-occurrence patterns. Ecology, 81, 2606-2621.

Gotelli, N.J. \& McCabe, D.J. (2002) Species co-occurrence: a meta-analysis of J. M. Diamond's assembly rules model. Ecology, 83, 2091-2096.

Gotelli, N.J. \& Ulrich, W. (2010) The empirical Bayes approach as a tool to identify non-random species associations. Oecologia, 162, 463-477.

Gotelli, N.J. \& Ulrich, W. (2012) Statistical challenges in null model analysis. Oikos, 121, 171-180.

Götzenberger, L., de Bello, F., Bråthen, K.A. et al. (2012) Ecological assembly rules in plant communities approaches, patterns and prospects. Biological Reviews, 87, 111-127.

Gravel, D., Canham, C.D., Beaudet, M. \& Messier, C. (2006) Reconciling niche and neutrality: the continuum hypothesis. Ecology Letters, 9, 399-409.

Habeck, J. (1959) A phytosociological study of the upland forest communities in the central Wisconsin sand plain area. Wisconsin Academy of Science, Arts and Letters, 48, 31-48.

Horner-Devine, M.C., Silver, J.M., Leibold, M.A. et al. (2007) A comparison of taxon co-occurrence patterns for macro- and microorganisms. Ecology, 88, 1345-1353.

Hubbell, S.P. (2001) The unified neutral theory of species abundance and diversity. Princeton University Press, Princeton, NJ.

Krasnov, B.R., Pilosof, S., Stanko, M., Morand, S., KoralloVinarskaya, N.P., Vinarski, M.V. \& Poulin, R. (2014) Co-occurrence and phylogenetic distance in communities of mammalian ectoparasites: limiting similarity versus environmental filtering. Oikos, 123, 63-70.

Kucharik, C.J., Serbin, S.P., Vavrus, S., Hopkins, E.J. \& Motew, M.M. (2010) Patterns of climate change across Wisconsin from 1950 to 2006. Physical Geography, 31, 1-28.

Larsen, S. \& Ormerod, S.J. (2014) Anthropogenic modification disrupts species co-occurrence in stream invertebrates. Global Change Biology, 20, 51-60.

Li, D. \& Waller, D. (2015) Drivers of observed biotic homogenization in pine barrens of central Wisconsin. Ecology, 96, 10301041.

Miklós, I. \& Podani, J. (2004) Randomization of presenceabsence matrices: comments and new algorithms. Ecology, 85, 86-92.

Oksanen, J., Blanchet, F.G., Kindt, R. et al. (2013) Vegan: community ecology package. https://cran.r-project.org/web/ packages/vegan/index.html

Olden, J.D. \& Poff, N.L. (2003) Toward a mechanistic understanding and prediction of biotic homogenization. The American Naturalist, 162, 442-460.

Pärtel, M., Szava-Kovats, R. \& Zobel, M. (2011) Dark diversity: shedding light on absent species. Trends in Ecology and Evolution, 26, 124-128.

Pitta, E., Giokas, S. \& Sfenthourakis, S. (2012) Significant pairwise co-occurrence patterns are not the rule in the majority of biotic communities. Diversity, 4, 179-193.
R Core Team (2015) R: A Language and Environment for Statistical Computing. R Foundation for Statistical Computing, Vienna, Austria.

Rogers, D.A., Rooney, T.P., Olson, D. \& Waller, D.M. (2008) Shifts in southern Wisconsin forest canopy and understory richness, composition, and heterogeneity. Ecology, 89, 2482-2492.

Rogers, D.A., Rooney, T.P., Hawbaker, T.J., Radeloff, V.C. \& Waller, D.M. (2009) Paying the extinction debt in southern Wisconsin forest understories. Conservation Biology, 23, 14971506.

Rooney, T.P. (2008) Comparison of co-occurrence structure of temperate forest herb-layer communities in 1949 and 2000. Acta Oecologica, 34, 354-360.

Sfenthourakis, S., Tzanatos, E. \& Giokas, S. (2006) Species co-occurrence: the case of congeneric species and a causal approach to patterns of species association. Global Ecology and Biogeography, 15, 39-49.

Stone, L. \& Roberts, A. (1990) The checkerboard score and species distributions. Oecologia, 85, 74-79.

Suttle, K.B., Thomsen, M.A. \& Power, M.E. (2007) Species interactions reverse grassland responses to changing climate. Science, 315, 640-642.

Ulrich, W. \& Gotelli, N.J. (2013) Pattern detection in null model analysis. Oikos, 122, 2-18.

Veech, J.A. (2013) A probabilistic model for analysing species co-occurrence. Global Ecology and Biogeography, 22, 252-260.

Veech, J.A. (2014) The pairwise approach to analysing species co-occurrence. Journal of Biogeography, 41, 1029-1035.

Waller, D., Amatangelo, K., Johnson, S. \& Rogers, D. (2012) Wisconsin vegetation database - plant community survey and resurvey data from the Wisconsin plant ecology laboratory. Biodiversity and Ecology, 4, 255-264.

\section{SUPPORTING INFORMATION}

Additional supporting information may be found in the online version of this article at the publisher's web-site.

Appendix S1 Supporting figures.

Appendix S2 Results based on null models that maintain species occurrence totals but not species richness at each sample unit.

BIOSKETCHES

Daijiang $\mathbf{~} \mathbf{i}$ is a $\mathrm{PhD}$ candidate at the University of Wisconsin-Madison. He has interests in community assembly, functional and phylogenetic ecology, and species diversity and ecosystem functioning.

Donald M. Waller is an evolutionary ecologist with interests in long-term ecological changes and the drivers of those changes (www.botany.wisc.edu/waller/).

Editor: David Currie 